\title{
Current plateaus of nonadiabatic charge pump: Multiphoton assisted processes
}

\author{
Baigeng Wang, ${ }^{1}$ Jian Wang, ${ }^{1,2, *}$ and Hong Guo ${ }^{3}$ \\ ${ }^{1}$ Department of Physics, The University of Hong Kong, Pokfulam Road, Hong Kong, China \\ ${ }^{2}$ Institute of Solid State Physics, Chinese Academy of Sciences, Hefei, Anhui, China \\ ${ }^{3}$ Department of Physics, McGill University, Montreal, Quebec, Canada H3A 2T8
}

(Received 8 April 2003; revised manuscript received 30 June 2003; published 24 October 2003)

\begin{abstract}
We report a theoretical investigation of quantum parametric charge pump in the nonadiabatic regime at finite pumping frequency and finite driving amplitude. A general and closed-form expression for pumped current is derived in the form of a continuous fraction reflecting the multiphoton processes which are found to play an important role. Our nonperturbative theory predicts a remarkable plateau structure in the pumped current due to these multiphoton assisted processes in a double-barrier quantum well involving only a single pumping potential. A current reversal is found as the resonant level of the pump crosses the Fermi energy of the leads.
\end{abstract}

DOI: 10.1103/PhysRevB.68.155326

PACS number(s): 73.23.Ad, 73.40.Gk, 72.10.Bg

\section{INTRODUCTION}

A parametric charge pump is a device that drives the flow of a dc electric current by a time-dependent variation of two or more device parameters. The quantum parametric pump has received considerable attention both experimentally and theoretically. ${ }^{1-33} \mathrm{~A}$ very useful and clear transport theory on quantum parametric pump was provided by Brouwer ${ }^{1}$ for the adiabatic regime, i.e., in the $\omega \rightarrow 0$ limit where $\omega$ is the frequency of the driving force. This theory was built on the scattering matrix approach for coherent ac transport developed earlier by Büttiker, Thomas, and Prêtre. ${ }^{34}$ The adiabatic limit is relevant for situations of slow potential modulation so that electrons traverse the device almost without noticing the change of the potential landscape. The experimental data of Switkes et al. ${ }^{3}$ has demonstrated the operation of a quantum parametric pump in the adiabatic regime where electrons were pumped out quantum coherently.

For parametric quantum pumps working at higher frequency, electrons may sample a time-dependent potential landscape as they traverse the device. In this situation one needs to consider the physics beyond the adiabatic regime. So far several papers have been devoted to the understanding of nonadiabatic quantum pumps. Moskalets and Büttiker have formulated a Floquet scattering matrix theory ${ }^{30}$ which treats adiabatic and nonadiabatic quantum pumps on equal footing, i.e., using scattering matrices, so that an exact solution was obtained numerically for an oscillating doublebarrier pump structure. For mesoscopic samples with a discrete spectrum, Floquet scattering matrix theory predicts ${ }^{30}$ a sign reversal of the pumped current for a specific pumping frequency $\omega$. Polianski, Vavilov, and Brouwer considered fluctuations of charge transmitted through a parametric pump, also from a scattering matrix formalism with a scattering matrix that depends on two times, and analyzed the average and variance of the noise for an ensemble of chaotic quantum dots. ${ }^{25}$ In addition, two of the present authors considered the heat current in nonadiabatic pumps ${ }^{26}$ with a scattering matrix theory, following the earlier work of Avron et al. that a pump is optimal if the heat current equals the power of Joule heat. ${ }^{21}$ While considerable understanding of nonadiabatic quantum pumps was established by these and other contributions, ${ }^{27}$ previous works ${ }^{25-27}$ were mostly based on perturbative schemes either in term of pumping frequency or pumping amplitude.

The purpose of this work is to develop a nonperturbative theory for quantum parametric pump in general terms of pumping frequency and pumping amplitude. Such a theory is useful for providing further understanding of charge pumps in the nonadiabatic regime, and helps to establish a more general and unambiguous physical picture of transport features not accessible by perturbative theories. We found that such a theory can be achieved by the Keldysh nonequilibrium Green's-function (NEGF) (Ref. 27) formalism. In particular, we derived a general formula of pumped current which, in various limits, reproduces known results of previous work where the driving parameter has a single sinusoidal mode. In the nonadiabatic regime, we predict transport features using an example of quantum-well charge pump that is driven by a single parameter: such a pump is only possible in the nonadiabatic regime. ${ }^{27}$ In particular, if the pumping potential is located at the barriers of the quantum well, the pumped current exhibits remarkable plateaus due to a multiphoton assisted pumping process. The width of a plateau is found to be determined by $\hbar \omega$ while its height varies depending on the pumping amplitude and coupling strength between the pump and the leads. By tuning a gate voltage that shifts the resonance level of the quantum well to cross the Fermi energy of the leads, we observe a direction reversal of the pumped current. We also investigate the pumped current when the single pumping parameter is located in the interior of the quantum well. We find that the pumped current increases rapidly by several orders of magnitude as the pumping parameter is shifted away from the symmetry center of the quantum well.

The rest of the paper is organized as follows. In the following section, we present the detailed derivation of the pumped current in the nonadiabatic regime with arbitrary pumping amplitude. Section III presents results for the quantum-well pump, and the last section is a short summary.

\section{THEORY}

In this section we derive the expression of pumped current in the nonadiabatic regime for an arbitrary mesoscopic 
conductor. We first cast the pumped current into a wellknown form in terms of retarded and advanced Green's functions, and then derive these Green's functions for the pump for arbitrary pumping frequency $\omega$ and pumping amplitude.

Consider a mesoscopic parametric pump which is defined by a Hamiltonian

$$
\hat{\mathbf{H}}=\hat{\mathbf{H}}_{d}+H^{\prime}(t),
$$

where $\hat{\mathbf{H}}_{d}$ describes the electronic structure of the pump, its leads, as well as the coupling between them. $H^{\prime}(t)$ is a timedependent driving potential for the quantum pump which we assume to have the following form:

$$
H^{\prime}(t)=(V / 2) \exp (i \omega t)+\left(V^{*} / 2\right) \exp (-i \omega t),
$$

where $V$ is the effective pumping potential profile. ${ }^{35}$ In the following, we neglect interaction between electrons in the ideal leads labeled by $\alpha \equiv L, R$.

The electron current operator in lead $\alpha$ can be evaluated from the Heisenberg equation of motion $\hat{I}_{\alpha}=d \hat{N}_{\alpha} / d t=$ $-i / \hbar\left[\hat{N}_{\alpha}, \hat{\mathbf{H}}\right]$, where $\hat{N}_{\alpha}$ is the number operator in lead $\alpha$, $[\cdots]$ denotes commutator, and $\hbar$ is the reduced Planck constant. Following Ref. 36, using a second quantized form of $\hat{\mathbf{H}}$ to carry out the commutator, the time-averaged current (setting $\hbar=1$ ) can be expressed in terms of Keldysh nonequilibrium Green's functions as follows: ${ }^{36}$

$$
\begin{aligned}
I_{\alpha}= & -\frac{q}{\tau} \int_{0}^{\tau} d t \int d t_{1} \operatorname{Tr}\left[\mathbf{G}^{r}\left(t, t_{1}\right) \mathbf{\Sigma}_{\alpha}^{<}\left(t_{1}, t\right)\right. \\
& \left.+\mathbf{G}^{<}\left(t, t_{1}\right) \mathbf{\Sigma}_{\alpha}^{a}\left(t_{1}, t\right)+\text { c.c. }\right],
\end{aligned}
$$

where $\tau=2 \pi / \omega$ is the period of pumping cycle; $G^{r, a}\left(t, t_{1}\right)$ are the retarded $(r)$ and advanced $(a)$ Green's functions; $\mathbf{G}^{<}$ is the lesser Green's function; and $\boldsymbol{\Sigma}_{\alpha}^{<}=i \Gamma_{\alpha} f_{\alpha}$ is the lesser self-energy which is determined by the linewidth function $\Gamma_{\alpha}$ and the Fermi function $f_{\alpha}$ of the $\alpha$ th lead. We now cast Eq. (3) into a well-known form in terms of retarded and advanced Green's functions.

Carrying out a double-time Fourier transform

$$
G^{\gamma}\left(t_{1}, t_{2}\right)=\int \frac{d E_{1}}{2 \pi} \frac{d E_{2}}{2 \pi} e^{-i E_{1} t_{1}+i E_{2} t_{2}} G^{\gamma}\left(E_{1}, E_{2}\right)
$$

with $\gamma=r, a,<$, Eq. (3) becomes

$$
\begin{aligned}
I_{\alpha}= & -\frac{q}{2 N \tau} \int \frac{d E_{1}}{2 \pi} \frac{d E_{2}}{2 \pi} \operatorname{Tr}\left\{\left[G^{r}\left(E_{1}, E_{2}\right)\right.\right. \\
& \left.-G^{a}\left(E_{1}, E_{2}\right)\right] \Sigma_{\alpha}^{<}\left(E_{2}, E_{1}\right)+G^{<}\left(E_{1}, E_{2}\right)\left[\Sigma_{\alpha}^{a}\left(E_{2}, E_{1}\right)\right. \\
& \left.\left.-\Sigma_{\alpha}^{r}\left(E_{2}, E_{1}\right)\right]\right\} \\
\equiv & -\frac{q}{8 N \tau \pi^{2}} \operatorname{Tr}\left[\left(\mathbf{G}^{r}-\mathbf{G}^{a}\right) \mathbf{\Sigma}_{L}^{<}+\mathbf{G}^{<}\left(\boldsymbol{\Sigma}_{L}^{a}-\mathbf{\Sigma}_{L}^{r}\right)\right]
\end{aligned}
$$

where we have extended the integration range for $d t$ in Eq. (3) to $[-N \tau, N \tau]$ with $N \rightarrow \infty$. Note that in Eq. (4) the matrix $\mathbf{G}^{\gamma}$ has element $G^{\gamma}\left(E_{1}, E_{2}\right)$. It is also easy to prove that
$\mathbf{G}^{r}-\mathbf{G}^{a}=-i \mathbf{G}^{r} \boldsymbol{\Gamma} \mathbf{G}^{a}$ where $\boldsymbol{\Gamma}=\sum_{\alpha} \boldsymbol{\Gamma}_{\alpha}$ is the total linewidth. Using the Keldysh equation $\mathbf{G}^{<}=\mathbf{G}^{r} \boldsymbol{\Sigma}^{<} \mathbf{G}^{a}$, and the fact that $\Sigma^{\gamma}\left(E_{2}, E_{1}\right)=2 \pi \delta\left(E_{1}-E_{2}\right) \Sigma^{\gamma}\left(E_{1}\right)$ at zero external bias, we obtain

$$
\begin{aligned}
I_{\alpha}= & \frac{q}{2 N \tau} \int \frac{d E_{1}}{2 \pi} \frac{d E_{2}}{2 \pi} \operatorname{Tr}\left[\Gamma_{\alpha}\left(E_{1}\right) G^{r}\left(E_{1}, E_{2}\right) \Gamma\left(E_{2}\right)\right. \\
& \left.\times G^{a}\left(E_{2}, E_{1}\right)\right]\left[f\left(E_{2}\right)-f\left(E_{1}\right)\right] .
\end{aligned}
$$

Finally, using the identity $\Sigma_{E}=2 N \tau \int d E /(2 \pi)$, we arrive at the well-known expression, ${ }^{22-26,30}$

$$
\begin{aligned}
I_{\alpha}= & \frac{q}{(2 N \tau)^{2}} \int \frac{d E}{2 \pi} \sum_{n=-\infty}^{+\infty} \operatorname{Tr}\left[\Gamma_{\alpha}(E) G^{r}(E, E+n \omega)\right. \\
& \left.\times \Gamma_{n}(E) G^{a}(E+n \omega, E)\right]\left[f_{n}(E)-f(E)\right],
\end{aligned}
$$

where $\Gamma_{n}(E) \equiv \Gamma(E+n \omega)$ and $f_{n}(E) \equiv f(E+n \omega)$. In this expression, the summation over integer $n$ clearly indicates the contributions from multiphoton processes toward the pumped current.

In the following, using Eq. (5) as the starting point and deriving the Green's functions for the pumping potential Eq. (2), we derive a closed-form solution for the pumped current $I_{\alpha}$.

\section{A. The retarded Green's function}

We now proceed to calculate $G^{r}(E, E+n \omega)$ which is needed for evaluating the pumped current through Eq. (5). $G^{r}$ is calculated by the Dyson equation which, after a double-time Fourier transform, becomes

$$
\begin{aligned}
G^{r}\left(E_{1}, E_{2}\right)= & 2 \pi G^{0 r}\left(E_{1}\right) \delta\left(E_{1}-E_{2}\right)+\int \frac{d E}{2 \pi} \\
& \times G^{r}\left(E_{1}, E_{2}+E\right) H^{\prime}(E) G^{0 r}\left(E_{2}\right),
\end{aligned}
$$

where $G^{0 r}$ is the equilibrium Green's function when the pumping potential $H^{\prime}$ is set to zero, and $H^{\prime}(E)=\pi[V \delta(E$ $\left.+\omega)+V^{*} \delta(E-\omega)\right]$ is the Fourier transform of $H^{\prime}(t)$ in Eq. (2). From now on we assume that the equilibrium Green's function $G^{0 r}$ has been known or calculated for the device Hamiltonian $\hat{\mathbf{H}}_{d}$ of Eq. (1), and an explicit example will be given in the following section.

Carrying out the energy integration in Eq. (6), we obtain

$$
\begin{aligned}
G^{r}\left(E_{1}, E_{2}\right)= & 2 \pi G^{0 r}\left(E_{1}\right) \delta\left(E_{1}-E_{2}\right)+\left[G^{r}\left(E_{1}, E_{2}-\omega\right) V\right. \\
& \left.+G^{r}\left(E_{1}, E_{2}+\omega\right) V^{*}\right] G^{0 r}\left(E_{2}\right) / 2
\end{aligned}
$$

We now use Eq. (7) to derive a general expression for $G^{r}(E, E+n \omega)$ as follows. We simplify notation using the following abbreviations: $G_{n}^{r}(E) \equiv G^{r}(E, E+n \omega)$ and $G_{n}^{0 r}(E) \equiv G^{0 r}(E+n \omega)$. Therefore, for example, Eq. (7) gives the multiphoton Green's function for $n=0, \pm 1$ as

$$
\begin{gathered}
G_{0}^{r}=2 \pi G_{0}^{0 r} \delta(0)+\left(G_{1}^{r} V^{*}+G_{-1}^{r} V\right) G_{0}^{0 r} / 2, \\
G_{1}^{r}=\left(G_{2}^{r} V^{*}+G_{0}^{r} V\right) G_{1}^{0 r} / 2,
\end{gathered}
$$




$$
G_{-1}^{r}=\left(G_{0}^{r} V^{*}+G_{-2}^{r} V\right) G_{-1}^{0 r} / 2 .
$$

Therefore, to compute $G_{0}^{r}$ one needs $G_{ \pm 1}^{r}$ which in turn needs $G_{ \pm 2}^{r}$, etc. Similarly, the multiphoton Green's function $G_{n}^{r}$ can be obtained by iterating the following expression obtained from Eq. (7):

$$
G_{n}^{r}=\left(G_{n+1}^{r} V^{*}+G_{n-1}^{r} V\right) G_{n}^{0 r} / 2 .
$$

This equation has the following recursive solution:

$$
G_{n}^{r}=G_{n-1}^{r} V G_{n}^{0 r} \frac{1}{2 \alpha_{n}^{r}},
$$

where $\alpha_{n}^{r}=\left[\left(a_{n}, \bar{a}_{n}\right),\left(a_{n+1}, \bar{a}_{n+1}\right),\left(a_{n+2}, \bar{a}_{n+2}\right), \ldots\right]$ with $a_{n} \equiv i V G_{n+1}^{0 r} / 2$ and $\bar{a}_{n}=i V^{*} G_{n}^{0 r} / 2$. The continued fraction $\left[\left(a_{1}, \bar{a}_{1}\right),\left(a_{2}, \bar{a}_{2}\right),\left(a_{3}, \bar{a}_{3}\right), \ldots\right]$ is defined as

$$
\begin{aligned}
& {\left[\left(a_{1}, \bar{a}_{1}\right),\left(a_{2}, \bar{a}_{2}\right), \ldots\right]}
\end{aligned}
$$

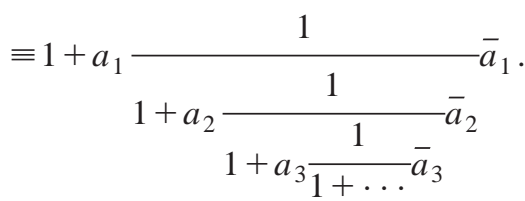

Since the equilibrium Green's functions $G_{n}^{0 r} \equiv G^{0 r}(E+n \omega)$ for the device Hamiltonian $\hat{\mathbf{H}}_{d}$ [see Eq. (1)] is assumed to be known, the continued fraction can be calculated to arbitrary order.

Setting $n= \pm 1$ in Eq. (9), we obtain $G_{1}^{r}$ $=G_{0}^{r} V G_{1}^{0 r} /\left(2 \alpha_{1}^{r}\right)$ and $G_{-1}^{r}=G_{0}^{r} V^{*} G_{-1}^{0 r} /\left(2 \beta_{-1}^{r}\right)$. Here $\beta_{-n}^{r}=\left[\left(b_{-n}, \bar{b}_{-n}\right),\left(b_{-n-1}, \bar{b}_{-n-1}\right), \ldots\right] \quad$ with $b_{-n}$ $=i V^{*} G_{-n-1}^{0 r} / 2$ and $\bar{b}_{-n}=i V G_{-n}^{0 r} / 2$. Similar to above, the continued fraction $\beta_{-n}^{r}$ is calculable to arbitrary order. Substituting these expressions of $G_{ \pm 1}^{r}$ into Eq. (8) and solving for $G_{0}^{r}(E)$, we obtain

$$
G_{0}^{r}(E)=\frac{2 \pi \delta(0)}{\left[G_{0}^{0 r}(E)\right]^{-1}-\Sigma^{r}(E)}
$$

with the multiphoton self-energy

$$
\Sigma^{r}=V G_{1}^{0 r} \frac{1}{4 \alpha_{1}^{r}} V^{*}+V^{*} G_{-1}^{0 r} \frac{1}{4 \beta_{-1}^{r}} V .
$$

Equation (11) provides the closed-form solution of multiphoton Green's function $G_{0}^{r}(E)$ which treats multiphoton processes exactly because its right-hand side includes arbitrary order of multiphoton processes through the continued fraction. Note that the factor $[2 \pi \delta(0)]^{2}$ in $G^{r}$ and $G^{a}$ cancels the factor $(2 N \tau)^{2}$ in Eq. (5).

Equations (9), (11), and (12) form the central results of this paper, they provide a closed-form solution of the parametric pumping in general terms of pumping frequency and amplitudes. The practical procedure is as follows. For a given device Hamiltonian $\hat{\mathbf{H}}_{d}$ [see Eq. (1)] which represents an arbitrary confining potential for the quantum pump, we calculate its retarded Green's function $G_{n}^{0 r}$. From $G_{n}^{0 r}$ we calculate $\Sigma^{r}$ from Eq. (12) and then evaluate Eq. (11) to obtain $G_{0}^{r}(E)$. Once $G_{0}^{r}$ is obtained, $G_{n}^{r}$ can be calculated recursively from Eq. (9) and the pumped current is then obtained from Eq. (5).

Although there is an infinite summation in Eq. (5) for the pumped current, because $G^{r}(E, E+n \omega) \equiv G_{n}^{r}$ is at least of the order of $V^{n}$ [see Eq. (9)], this summation converges rapidly even in the strong pumping regime as we will show with an example in the following section. We also note that continued fractions such as Eq. (10), which appears in our formula, are rather typical in describing multiphoton scattering process. ${ }^{36}$ Finally, using the fact that $\mathbf{G}^{r} \boldsymbol{\Gamma} \mathbf{G}^{a}=\mathbf{G}^{a} \boldsymbol{\Gamma} \mathbf{G}^{r}$, it is not difficult to prove that $\Sigma_{\alpha} I_{\alpha}=0$, i.e., current conservation for parametric pumping is satisfied by our results.

\section{B. Limiting cases}

In this section we analyze two known limiting cases of parametric quantum pumping using our results Eqs. (9), (11), and (12), together with the starting expression Eq. (5).

The first case concerns a general $\omega$ but small pumping amplitude $V$. In Refs. 27,37 the pumped current was obtained using a perturbation theory up to the second order in pumping amplitude $V$ at arbitrary pumping frequency $\omega$. Because our $G_{n}^{r} \sim V^{n}$ [see Eq. (9)] and the pumped current $I_{\alpha}$ $\sim G_{n}^{r} G_{n}^{a}$, the perturbative result is recovered from the general expressions (9), (11), and (12) by only keeping terms with $n= \pm 1$. Therefore, from Eq. (8) and neglecting $G_{ \pm 2}^{r}$ terms, we obtain $G_{1}^{r} \approx G_{0}^{0 r} V G_{1}^{0 r} / 2$. Equation (5) is now reduced to

$$
I_{\alpha}=q \int \frac{d E}{8 \pi} \sum_{n= \pm 1} \operatorname{Tr}\left[\Gamma_{\alpha} G_{0}^{0 r} V G_{n}^{0 r} \Gamma_{n} G_{n}^{0 a} V^{*} G_{0}^{0 a}\right]\left(f_{n}-f\right),
$$

which is exactly the same as that found in Ref. 27.

As a second limiting case, we show that Eqs. (9), (11), and (12) give the well-known result for the adiabatic regime. ${ }^{1}$ The adiabatic regime of parametric pumping concerns the $\omega \rightarrow 0$ limit. To take this limit, we apply the instantaneous approximation for the Green's function. We transform Eq. (5) to the Wigner representation using $G^{r, a}\left(t_{1}, t_{2}\right)$ $=\int(d E / 2 \pi) e^{-i E\left(t_{1}-t_{2}\right)} \mathcal{G}^{r, a}(E, T)$ where $T \equiv\left(t_{1}+t_{2}\right) / 2$ and $\mathcal{G}^{r, a}$ are the Green's functions in the Wigner representation. We then obtain ${ }^{38}$

$$
G_{n}^{r}=\int d T \mathcal{G}^{r}(E, T) e^{-i n \omega T} .
$$

Substituting this equation into Eq. (5) and keeping only the $O(\omega)$ term, we obtain

$$
\begin{aligned}
I_{\alpha}= & \frac{q}{(2 N \tau)^{2}} \int \frac{d E}{2 \pi} \int_{-\infty}^{\infty} d T d T^{\prime} \sum_{n=-\infty}^{+\infty} n \omega \\
& \times \operatorname{Tr}\left[\Gamma_{\alpha}(E) \mathcal{G}^{r}(E, T) \Gamma(E) \mathcal{G}^{a}\left(E, T^{\prime}\right)\right] \\
& \times \exp \left[-i n \omega\left(T-T^{\prime}\right)\right] \partial_{E} f(E) .
\end{aligned}
$$

Note that 


$$
\begin{aligned}
& \int d T \sum_{n=-\infty}^{+\infty} n \omega \mathcal{G}^{r}(E, T) \exp \left[-i n \omega\left(T-T^{\prime}\right)\right] \\
& =-i \int d T \partial_{T^{\prime}} \mathcal{G}^{r}(E, T) \sum_{n=-\infty}^{+\infty} \exp \left[-i n \omega\left(T-T^{\prime}\right)\right] \\
& =-2 i N \tau \partial_{T^{\prime}} \mathcal{G}^{r}\left(E, T^{\prime}\right),
\end{aligned}
$$

where we have used the identity $\Sigma_{E}=2 N \tau \int d E /(2 \pi)$. Equation (13) now becomes

$$
\begin{aligned}
I_{\alpha}= & \frac{-i q}{\tau} \int_{0}^{\tau} d T \int \frac{d E}{2 \pi} \operatorname{Tr}\left[\Gamma_{\alpha}(E) \partial_{T} \mathcal{G}^{r}(E, T) \Gamma(E)\right. \\
& \left.\times \mathcal{G}^{a}(E, T)\right] \partial_{E} f(E) .
\end{aligned}
$$

Using the Fisher-Lee relation ${ }^{39}$

$$
S_{\alpha \beta}=\delta_{\alpha \beta}-\Gamma_{\alpha}^{1 / 2} \mathcal{G}^{r} \Gamma_{\beta}^{1 / 2}
$$

we obtain

$$
\begin{gathered}
\partial_{T} S_{\alpha \beta}=-\Gamma_{\alpha}^{1 / 2} \partial_{T} \mathcal{G}^{r} \Gamma_{\beta}^{1 / 2}, \\
S_{\alpha \beta}^{\dagger}=-\Gamma_{\alpha}^{1 / 2} \partial_{T} \mathcal{G}^{a} \Gamma_{\beta}^{1 / 2} .
\end{gathered}
$$

Therefore, Eq. (14) becomes

$$
\begin{aligned}
I_{\alpha}= & \frac{-i q}{\tau} \int_{0}^{\tau} d T \int \frac{d E}{2 \pi} \sum_{\beta} \operatorname{Tr} \\
& \times\left[\partial_{T} S_{\alpha \beta}(E, T) S_{\alpha \beta}^{\dagger}(E, T)\right] \partial_{E} f(E),
\end{aligned}
$$

with $\tau=2 \pi / \omega$. Equation (15) is exactly the same as the formula derived in Ref. 30 [formula (18) of this reference], which has been shown ${ }^{30}$ to be equivalent to Brouwer's expression ${ }^{1}$ of adiabatic pumping.

\section{EXAMPLE: SINGLE PARAMETER DOUBLE- BARRIER QUANTUM PUMP}

The closed-form solution of quantum parametric pumping at arbitrary $\omega$ and amplitude $V$, Eqs. (9), (11), and (12) together with Eq. (5), is applicable to pump structures involving arbitrary confining potentials described by the device Hamiltonian $\hat{\mathbf{H}}_{d}$ [see Eq. (1)] whose retarded Green's function $G^{0 r}$ was assumed to be known in the previous derivations.

In this section we give an example of a quantum parametric pump whose device Hamiltonian $\hat{\mathbf{H}}_{d}$ is a one-dimensional (1D) symmetric double $\delta$-barrier potential,

$$
U(x)= \begin{cases}V_{0} \delta(x+a)+V_{0} \delta(x-a) & \text { if }-a<x<a \\ 0 & \text { otherwise, }\end{cases}
$$

where $2 a$ is the $1 \mathrm{D}$ quantum-well width and $V_{0}$ the barrier height. In the adiabatic theory (the $\omega \rightarrow 0$ limit), two pumping potentials are needed in order to give a nonzero pumped current. ${ }^{1}$ At a finite frequency, a single pumping potential appears to be enough to pump a dc current ${ }^{27}$ which is rather

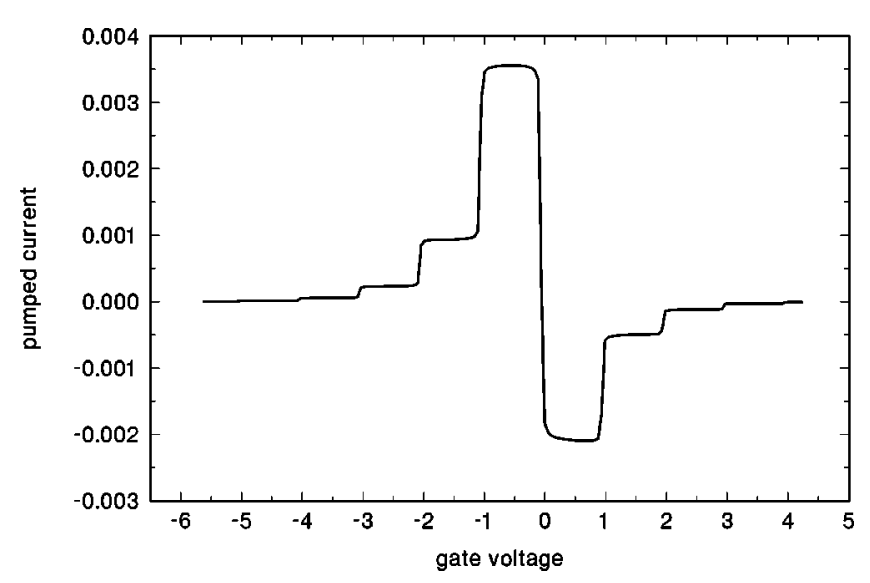

FIG. 1. The pumped current vs the gate voltage for $x_{0}=-a$, $\omega=1$, and $v_{p}=160$. We have set $n=20$ in the calculation.

peculiar. The nonperturbative theory presented above allows us to investigate this situation clearly and unambiguously. Therefore, in this example the driving potential of the quantum pump $H^{\prime}(t)$ [see Eq. (1)] is a single sinusoidal potential, $H^{\prime}(t) \equiv V(x, t)=V_{p} \cos (\omega t) \delta\left(x-x_{0}\right)$ with a real amplitude $V_{p} . x_{o}$ is the space point where $H^{\prime}(t)$ is applied. We calculate the pumped current in the left lead [setting $\alpha=L$ in Eq. (5)] at zero temperature. A gate voltage $v_{g}$ is applied to the double-barrier structure to tune the resonant single-particle energy level $\epsilon_{o}$ in the quantum well. We fix the Fermi level of leads at $E_{f}=0$ and assume it aligns with the resonant level $\epsilon_{o}$ when $v_{g}=0$. In the following, the unit is set by $\hbar=2 \mathrm{~m}$ $=q=2 a=1$. For GaAs material with $a=1000 \AA$, the energy unit is $E=0.056 \mathrm{meV}$ which corresponds to $\omega$ $=13.2 \mathrm{GHz}$. The unit for pumped current becomes 5 $\times 10^{-10}$ A. Finally, we set the double-barrier height $V_{0}$ $=200$ unless specified otherwise.

For the double-barrier confining potential Eq. (16), the equilibrium Green's function $G^{0 r}$ has been calculated exactly in Ref. 40 by solving the Dyson equation (6). Using this $G^{0 r}$ [see Eq. (3) of Ref. 40], we evaluate Eq. (12) followed by Eqs. (11) and (9). Finally we evaluate Eq. (5) for the pumped current.

Figure 1 shows the pumped current versus gate voltage in the strong pumping regime for $V_{p}=160$ and $\omega=1$. Here we applied the pumping driving force $H^{\prime}(t)$ at the left barrier, i.e., we set $x_{0}=-a$. The following observations are in order.

(1) The pumped current displays a series of remarkable "plateaus." The width of a plateau is equal to $\hbar \omega$ whereas the height of the plateau depends on $V_{p}$ and $V_{0}$ in a nonlinear fashion.

(2) The pumped current reverses its direction when the resonant level crosses the Fermi level of the lead, i.e., when $v_{g}$ changes sign. As a result, we see from Fig. 1 that the pumped current is quenched near $v_{g}=0$.

(3) The height of the current plateaus for negative $v_{g}$ is considerably larger than those of positive $v_{g}$.

Why the pumped current has a plateau structure? To understand this, we rewrite Eq. (5) exactly into the following form: 


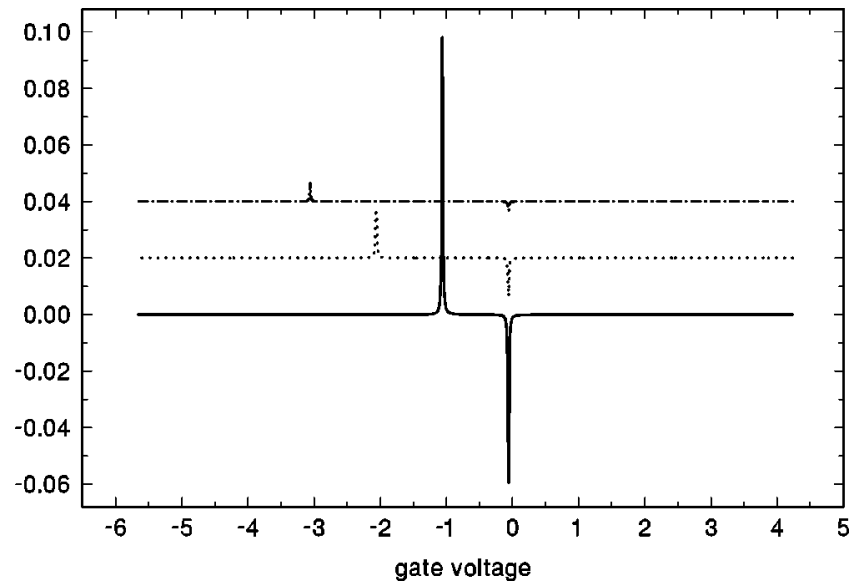

FIG. 2. The integrand of Eq. (17) vs gate voltage for $n=1$ (solid line), $n=2$ (dotted line), and $n=3$ (dash-dotted line). The system parameters are the same as that of Fig. 1. For illustrating purpose, we have offset the curve by 0.02 .

$$
I_{L}=q \int \frac{d E}{2 \pi} \sum_{n=1}^{+\infty} F_{n}(E)\left[f_{n}(E)-f(E)\right]
$$

with

$$
\begin{aligned}
F_{n}(E)= & \frac{q}{(2 N \tau)^{2}} \operatorname{Tr}\left[\Gamma_{L}(E) G^{r}(E, E+n \omega) \Gamma_{n}(E)\right. \\
& \times G^{a}(E+n \omega, E)-\Gamma_{L n}(E) G^{r}(E+n \omega, E) \Gamma(E) \\
& \left.\times G^{a}(E, E+n \omega)\right] .
\end{aligned}
$$

We begin by recalling two important facts. First, although the summation over photon number $n$ goes to $\infty$, only a few terms with small $n$ have significant contributions to current as discussed above. Second, although the energy integration is from $-\infty$ to $+\infty$, the Fermi functions in Eq. (17) cut this range to $[0, n \omega]$ at low temperatures. Next, we note that the kernel $F_{n}$ consists of two terms, one due to photon absorption process $^{41}$ and the other to photon emission process. These two contributions differ by a sign indicating a competition between photon absorption and emission. ${ }^{27} F_{n}$ has sharp features due to resonance tunneling: as we tune the gate voltage $v_{g}$ to align the energy level $\epsilon_{o}$ of the quantum well to the Fermi level of the leads $\left(E_{f}=0\right), F_{n}$ shows a sharp feature. $F_{n}$ also shows sharp features at integer numbers of frequency. For example, for a negative $v_{g}$ which pulls $\epsilon_{o}$ to below $E_{f}$ such that $E_{f}-\epsilon_{o}=n \omega$, an electron can tunnel into level $\epsilon_{o}$, absorb one or more photons to gain energy $n \omega$, and tunnel out of the pump. For positive $v_{g}$ it is the photon emission processes that are relevant. The sidebands in $F_{n}(E)$ (Fig. 2) due to photon absorption and photon emission are clearly seen [for an $n$-photon emission process, the energy has been shifted by $n \omega$ due to the transformation from Eq. (5) to Eq. (17)]. The pumped current is the energy integration of $F_{n}(E)$ in the energy window $E=[0, n \omega]$ according to Eq. (17). Because for a given range of $v_{g}, F_{n}(E)$ has only a fixed number of peaks/dips in the range $E$ $=[0, n \omega]$, therefore from Eq. (17) the current is a constant

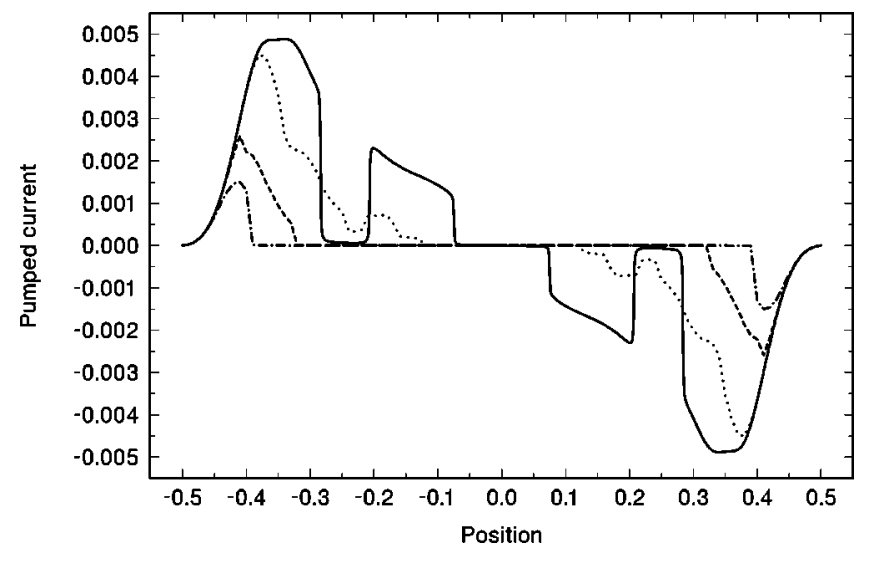

FIG. 3. The pumped current vs position of the pumping potential. Here $v_{g}=-0.67, \omega=1$, and $v_{p}=6$. In the calculation, we have set $n=20$ (solid line), $n=5$ (dotted line), $n=2$ (dashed line), and $n=1$ (dot-dashed line).

for this range of $v_{g}$ hence a current plateau is obtained in the $I_{\alpha}$ versus $v_{g}$ curve (see Fig. 1). We conclude that the plateau structure is a direct result of multiphoton assisted processes.

For the above example where the pumping force is on the left barrier $\left(x_{0}=-a\right)$, the positive current is due to photon absorption processes and the current reverses its sign if photon emission process dominates. The behavior of current reversal has been observed before for pumping in carbon nanotubes, ${ }^{42}$ charge quantization, ${ }^{18}$ and heat current generated during the pumping, ${ }^{30}$ although from completely different origins. Due to the energy dependence of coupling between the pump and the leads, the sideband is asymmetric with a larger peak for photon absorption process. As a result, the height of the current plateau is larger for photon absorption process. Our analysis showed that this plateau structure persists for different frequencies and pumping amplitudes. Further analysis also shows that there are two effects influencing the current plateau structure. The first is the barrier height of the quantum well: current plateaus can only be observed in the strong tunneling regime and they disappear for low barrier height. The second is temperature: the plateau structure is rounded off at finite temperature and is destroyed when temperature reaches $\sim 200 \mathrm{mK}$ for $\omega=10 \mathrm{GHz}$.

In the following, we investigate the situation when the pumping potential is inside the double-barrier structure, i.e., when $-a<x_{0}<a$. For this situation, we found a similar plateau structure in the pumped current due to the same photon assisted processes as discussed in the last paragraph. In Fig. 3 , the pumped current as a function of pumping position $x_{0}$ is plotted at a fixed $v_{g}=-0.67$ for a much smaller pumping amplitude $v_{p}=6$. In the calculation, we have included the contributions from one photon (dot-dashed line), two photons (dashed line), five photons (dotted line), and twenty photons (solid line). We have checked the convergence by including thirty photons: the difference is within $10^{-8}$. We observe from Fig. 3 that the pumped current $I_{p}$ is antisymmetric about the center of the pump. As expected, for a symmetric structure $\left(x_{0}=0\right), I_{p}$ vanishes identically. At $x_{0}=$ -0.5 , we found $I_{p}=2 \times 10^{-6}$. Figure 3 indicates that $I_{p}$ can 


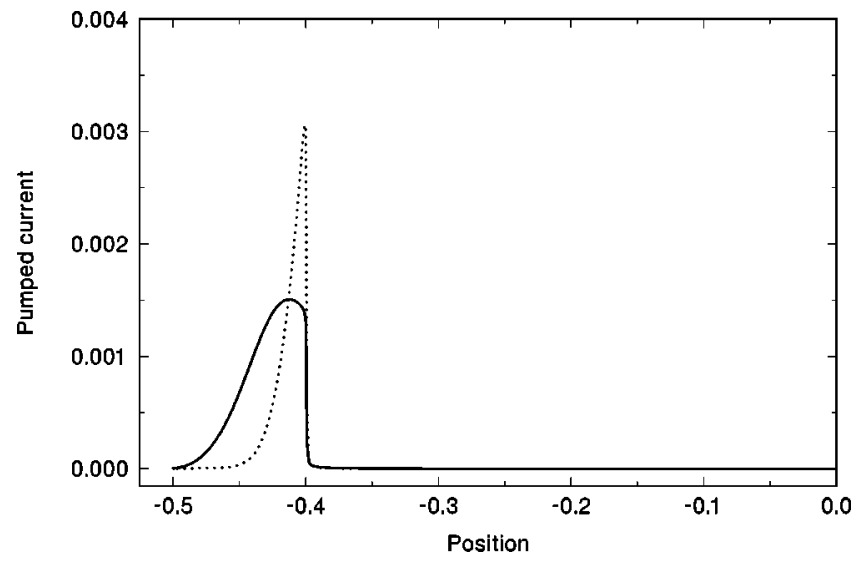

FIG. 4. The pumped current and the one-photon DOS vs position of the pumping potential. Here $v_{g}=-0.67, \omega=1$, and $v_{p}$ $=6$. For illustrating purpose, the one-photon DOS has been multiplied by 0.01 .

increase by three orders of magnitude by varying the position of the pumping potential away from the barriers.

Figure 3 shows abrupt changes of pumped current as $x_{0}$ is varied. To understand this, we investigate the one-photon process for which the pumped current is

$$
\begin{aligned}
I_{\alpha}= & \frac{q}{(8 N \tau)^{2}} \int \frac{d E}{2 \pi} \sum_{n= \pm 1} \operatorname{Tr}\left[G_{0}^{a} \Gamma_{\alpha} G_{0}^{r} V G_{n}^{0 r} \Gamma_{n} G_{n}^{0 a}\right] \\
& \times\left[f_{n}(E)-f(E)\right] .
\end{aligned}
$$

In Fig. 4, we plot the one-photon density of states (DOS) defined as

$$
\int \frac{d E}{2 \pi} \operatorname{Tr}\left[G_{0}^{a} \Gamma_{\alpha} G_{0}^{r}\right]\left[f_{1}(E)-f(E)\right],
$$

which is highly localized spatially with abrupt change as $x_{0}$ is varied, correlating very well with the abrupt variations in the pumped current. We conclude that the abrupt change in the pumped current as a function of position of the pumping potential is due to quantum interference of multiphoton assisted processes. Finally, the pumped current in Fig. 3 shows a plateau region when $-0.1<x<0.1$. Our numerical results show that the pumped current in that region is nonzero and is larger than the pumped current when the pumping potential is located at the barrier. As the strength of the pumping potential increases, the pumped current in the plateau region also increases.

\section{SUMMARY}

In this work, we have derived a general and nonperturbative expression for the electric current of parametric quantum pumps at finite frequency and finite pumping amplitude. The theory is also general enough so that arbitrary device structures can be investigated including situations where the pumping potential is located in the interior of the scattering region. We have shown that this theory reproduces previously known results in both adiabatic regime and in perturbative approach.

For a double-barrier quantum-well pump, we have shown that in the nonadiabatic regime, a single parameter is adequate to pump a dc current due to multiphoton processes. Such a current is found to have plateau structure precisely due to the multiphoton assisted processes. The width of the current plateau is determined by the pumping frequency. As a gate voltage is varied to shift the resonance level of the quantum well across the Fermi energy of leads, the pumped current is found to reverse its direction due to a competition of photon absorption and emission processes.

\section{ACKNOWLEDGMENTS}

We gratefully acknowledge support by a RGC grant from the SAR Government of Hong Kong under Grant No. HKU 7113/02P, a CRCG Grant from the University of Hong Kong, and from NSERC of Canada and FCAR of Quebec (H.G).
*Electronic address: jianwang@hkusub.hku.hk

${ }^{1}$ P.W. Brouwer, Phys. Rev. B 58, R10 135 (1998).

${ }^{2}$ I.L. Aleiner and A.V. Andreev, Phys. Rev. Lett. 81, 1286 (1998).

${ }^{3}$ M. Switkes, C. Marcus, K. Capman, and A.C. Gossard, Science 283, 1905 (1999).

${ }^{4}$ F. Zhou, B. Spivak, and B.L. Altshuler, Phys. Rev. Lett. 82, 608 (1999).

${ }^{5}$ T.A. Shutenko, I.L. Aleiner, and B.L. Altshuler, Phys. Rev. B 61, 10366 (2000).

${ }^{6}$ Y.D. Wei, J. Wang, and H. Guo, Phys. Rev. B 62, 9947 (2000).

${ }^{7}$ I.L. Aleiner, B.L. Altshuler, and A. Kamenev, Phys. Rev. B 62, 10 373 (2000).

${ }^{8}$ J.E. Avron, A. Elgart, G.M. Graf, and L. Sadun, Phys. Rev. B 62, R10 618 (2000).

${ }^{9}$ P.W. Brouwer, Phys. Rev. B 63, 121303 (2001).

${ }^{10}$ M. Moskalets and M. Buttiker, Phys. Rev. B 64, 201305 (2001).

${ }^{11}$ J. Wang et al., Appl. Phys. Lett. 79, 3977 (2001).

${ }^{12}$ F. Renzoni and T. Brandes, Phys. Rev. B 64, 245301 (2001).

${ }^{13}$ Y. Makhlin and A.D. Mirlin, Phys. Rev. Lett. 87, 276803 (2001).
${ }^{14}$ C.S. Tang and C.S. Chu, Solid State Commun. 120, 353 (2001).

${ }^{15} \mathrm{Y}$. Levinson, O. Entin-Wohlman, and P. Wolfle, cond-mat/0104408 (unpublished).

${ }^{16}$ J. Wang and B.G. Wang, Phys. Rev. B 65, 153311 (2002); B.G. Wang and J. Wang, ibid. 65, 233315 (2002).

${ }^{17}$ O. Entin-Wohlman, A. Aharony, and Y. Levinson, Phys. Rev. B 65, 195411 (2002).

${ }^{18}$ O. Entin-Wolman and A. Aharony, Phys. Rev. B 66, 035329 (2002).

${ }^{19}$ M. Blaauboer, Phys. Rev. B 65, 235318 (2002).

${ }^{20}$ Y. Levinson, O. Entin-Wohlman, and P. Wolfle, Physica A 302, 335 (2001).

${ }^{21}$ J.E. Avron, A. Elgart, G.M. Graf, and L. Sadun, Phys. Rev. Lett. 87, 236601 (2001).

${ }^{22}$ M.G. Vavilov, V. Ambegaokar, and I.L. Aleiner, Phys. Rev. B 63, 195313 (2001)

${ }^{23}$ V.I. Yudson, E. Kanzieper, and V.E. Kravtsov, Phys. Rev. B 64, 045310 (2001). 
${ }^{24}$ M. Moskalets and M. Buttiker, Phys. Rev. B 66, 035306 (2002).

${ }^{25}$ M.L. Polianski, M.G. Vavilov, and P.W. Brouwer, Phys. Rev. B 65, 245314 (2002).

${ }^{26}$ B.G. Wang and J. Wang, Phys. Rev. B 66, 125310 (2002); 66, 201305 (2002)

${ }^{27}$ B.G. Wang, J. Wang, and H. Guo, Phys. Rev. B 65, 073306 (2002).

${ }^{28}$ Y.D. Wei and J. Wang, Phys. Rev. B 66, 195419 (2002).

${ }^{29}$ J.L. Wu, B.G. Wang, and J. Wang, Phys. Rev. B 66, 205327 (2002)

${ }^{30}$ M. Moskalets and M. Buttiker, Phys. Rev. B 66, 205320 (2002).

${ }^{31}$ B.G. Wang, J. Wang, and H. Guo, Phys. Rev. B 67, 092408 (2003).

${ }^{32}$ Q.F. Sun, H. Guo, and J. Wang, Phys. Rev. Lett. 90, 258301 (2003); W. Long, Q.F. Sun, H. Guo, and J. Wang, Appl. Phys. Lett. 83, 1397 (2003).

${ }^{33}$ M. Moskalets and M. Buttiker, cond-mat/0302586 (unpublished).

${ }^{34}$ M. Büttiker, H. Thomas, and A. Prêtre, Z. Phys. B: Condens. Matter 94, 133 (1994); M. Büttiker, J. Phys.: Condens. Matter 5, 9361 (1993).
${ }^{35}$ Here $V$ is a diagonal matrix with matrix element $V_{x} \exp \left(i \phi_{x}\right)$ where $x$ labels the position, $V_{x}$ is real, and $\phi_{x}$ is the phase. For a single driving force located at site $x=x_{0}$ with pumping amplitude $V_{0}$, the only nonzero matrix element is $V_{0} \exp \left(i \phi_{0}\right) \delta_{x x_{0}}$ and the pumping potential becomes $H^{\prime}=V_{0} \cos \left(\omega t+\phi_{0}\right) \delta_{x x_{0}}$. For two pumping potentials at $x=x_{1}$ and $x_{2}$ with pumping amplitude $V_{0}$, the nonzero matrix element is $V_{0} \exp \left(i \phi_{1}\right) \delta_{x x_{1}}+V_{0} \exp \left(i \phi_{2}\right) \delta_{x x_{1}}$. The pumping potential $H^{\prime}=V_{0} \cos \left(\omega t+\phi_{1}\right)+V_{0} \cos \left(\omega t+\phi_{2}\right)$.

${ }^{36}$ A.P. Jauho, N.S. Wingreen, and Y. Meir, Phys. Rev. B 50, 5528 (1994).

${ }^{37}$ The result in Ref. 27 has also been obtained using scattering matrix in Ref. 24.

${ }^{38}$ Similar to Ref. 30 , we have used the adiabatic approximation so that the $\mathcal{G}^{r}(E+n \omega / 2, T) \approx \mathcal{G}^{r}(E, T)$.

${ }^{39}$ D.S. Fisher and P.A. Lee, Phys. Rev. B 23, 6851 (1981).

${ }^{40}$ M.K. Yip, J. Wang, and H. Guo, Z. Phys. B: Condens. Matter 104, 463 (1997).

${ }^{41}$ Since $G_{n}^{r}$ is the renormalized Green's function, it also has a photon emission component through the dependence of $\beta_{-n}^{r}$.

${ }^{42}$ Y. D. Wei et al., Phys. Rev. B 64, 115321 (2001). 